\title{
Application of Mathieu Functions and the Point Matching Method to Elliptic Conductors
}

\section{Wisnu Wurjantara}

Ryerson University

\section{Ali M. Hussein}

Ryerson University

Wurjantara, W., \& Hussein, A. M. (1997). Application of Mathieu functions and the point matching method to elliptic conductors. Proceedings of the 1997 IEEE International Magnetics Conference (INTERMAG), EP04.

doi:10.1109/INTMAG.1997.597692

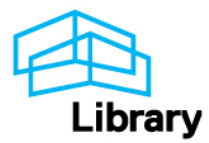




\section{APPLICATION OF MATHIEU FUNCTIONS AND THE POINT MATCHING} METHOD TO ELLIPTIC CONDUCTORS

Wisnu Wurjantara and Ali M. Hussein

Department of Electrical Engineering, Ryerson Polytechnic University, 350 Victoria St.,

Toronto, Ontario, M5B-2K3, Canada.

Introduction

Analysis of elliptic conductors, carrying a known total current, using the point matching method (PMM) in circular-cylinder coordinates [1] failed for the values of axes ratio $b / a<0.5$, where $a$ and $b$ are the major and minor axes of the ellipse, respectively. This work shows that the use of elliptic-cylinder coordinates in conjunction with the point matching method overcomes this problem. This paper is a part of the work investigating the difficulties encountered in the point matching method, and is motivated by the fact that, despite its limitations, the method is still attractive as suggested by its use in recent papers [2]-[4]

Analysis

Fig. 1 shows the cross section of a long elliptic conductor with a total current $I$ flowing in the $+z$ direction with a time dependence of $e^{j a x}$. The electric field in the conductor has only $z$-component, $E_{z}$. The wave equation that $E_{z}$ has to satisfy can be solved in ellipticcylinder coordinates $(\eta, \varphi)$ to yield the expressions for the fields inside $\left(\eta<\eta_{s}\right)$ and outside $\left(\eta>\eta_{\mathrm{s}}\right)$ the conductor:

$$
\begin{aligned}
& E_{z}=\sum_{n=0}^{\infty} C_{n} c e_{2 n}(\varphi) C e_{2 n}(\eta), \quad \eta<\eta_{s} \\
& E_{z}=\frac{j \omega \mu I}{2 \pi} \eta+\sum_{n=0}^{\infty} B_{n} \cos (2 n \varphi) e^{-2 n \eta}, \quad \eta>\eta_{s}
\end{aligned}
$$

where $c e_{2 n}(\varphi)$ 's are even, periodic Mathieu functions of order $2 n$, and $C e_{2 n}(\eta)$ 's are the corresponding modified Mathieu functions. The azimuthal magnetic field, $H_{\varphi}$, is found in both regions using Maxwell's equations:

$$
\begin{aligned}
& H_{\varphi}=\frac{1}{j \omega \mu \rho \sqrt{\cosh ^{2} \eta-\cos ^{2} \varphi}} \sum_{n=0}^{\infty} C_{n} c e_{2 n}(\varphi) C e_{2 n}^{\prime}(\eta), \eta<\eta_{s} \\
& H_{\varphi}=\frac{1}{j \omega \mu \rho \sqrt{\cosh ^{2} \eta-\cos ^{2} \varphi}}\left[\frac{j \omega \mu I}{2 \pi}-\sum_{n=0}^{\infty} 2 n B_{n} \cos (2 n \varphi) e^{-2 n n}\right], \eta>\eta_{s}
\end{aligned}
$$

\section{Ali M. Hussein}

Department of Electrical Engineering, Ryerson Polytechnic University

350 Victoria Street, Toronto, Ontario, M5B-2K3, Canada

Telephone: $979-5000$ ext 6108, fax: $979-5280$ where $\rho=\sqrt{a^{2}-b^{2}}$. The unknown coefficients $C_{n}$ 's and $B_{n}$ 's are found by matching the tangential electric and magnetic fields, $E_{z}$, and $H_{p}$, at selected points on the conductor's surface. Fig. 2 and Fig. 3 show the normalized magnitude and phase angle of $E_{2}$ on the conductor's surface for various values of axes ratio. For comparison, Fig. 4 shows the erroneous result obtained using the point matching method in circular-cylinder coordinates for $b / a=0.4$. All computations are carried out with $a / \delta=5$ where $\delta$ is the skin depth The distance $d$ is measured from point $\mathrm{A}$ in the direction of the arrow AB (Fig. 1).

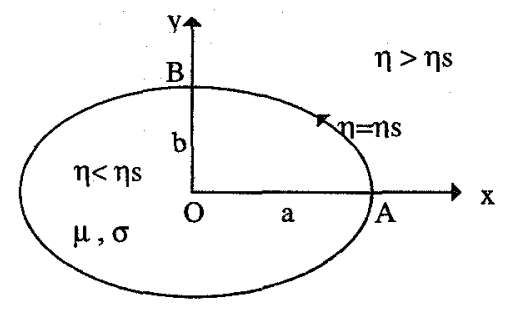

Fig.1. Cross section of an elliptic conductor

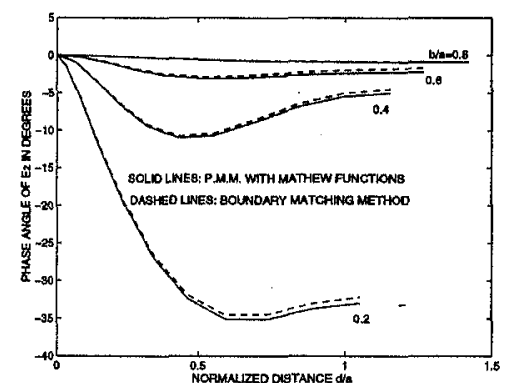

Fig. 3. Phase angle of $E_{z}$.

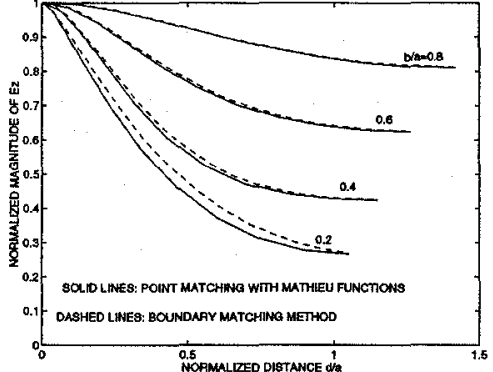

Fig. 2. Normalized magnitude of $E_{z}$.

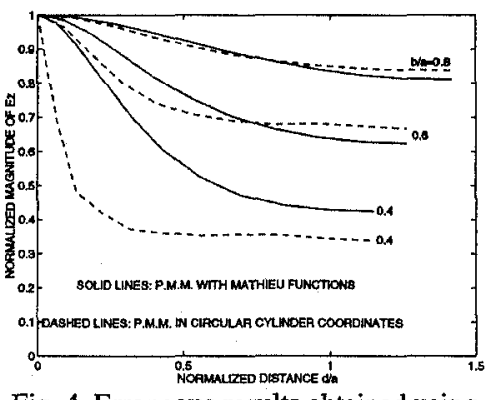

Fig. 4. Erroneous results obtained using PMM in circular-cylinder coordinates.

\section{References}

[1] A. M. Hussein and P. P. Biringer, J. Appl. Phys., vol. 60, 3356, (1986)

[2] A. Boag, Y. Leviatan, A. Boag, IEEE Trans. Ant. Propag. AP-41 926, (1993)

[3] H. Na and H. Kim, IEEE Trans. Ant. Propag. AP-43, 426 (1995).

[4] J. M. Tranquilla and H. M. Al-Rizzo, IEEE Trans. Ant. Propag. AP-43, 63, (1995). 